\title{
Feature Availability Comparison in Free and Paid Versions of Popular Smartphone Weight Management Applications
}

\author{
Telema Briggs, $\mathrm{BS}^{1}$; Virginia Quick, $\mathrm{PhD}, \mathrm{RD}^{1}$; William K. Hallman, $\mathrm{PhD}^{2}$
}

\begin{abstract}
Objective: Characterize capabilities of nutrition applications (apps) for weight management and associations between features, ratings, and app installations.

Design: Calorie tracking apps with weight management as a primary outcome were selected from the Apple App Store and Google Play Store using keywords "diet" and "weight loss."

Methods: Reviewers assessed free and upgraded versions of nutrition apps $(n=15)$ for features within 4 categories: (1) dietary intake, (2) anthropometrics, (3) physical activity, and (4) behavior change strategies. Outcome Measures: Presence of specific app features, app ratings, and app installations.

Analysis: Descriptive statistics of free and paid app versions. Spearman rank-order correlations were used to determine associations between feature inclusion, app ratings, and installations.

Results: The apps had the greatest number of features in the dietary intake category. Additional dietary intake features were those most likely obtained through a subscription purchase. Behavior change content was absent from most apps. The macronutrient adjustment feature was strongly associated with average app ratings $\left(r_{s}=0.74 ; P<0.002\right)$ and with subscription costs $\left(r_{s}=0.60 ; P<0.019\right)$.

Conclusions and Implications: This study found most nutrition apps possess an abundance of features dedicated to dietary intake, anthropometric, and physical activity tracking while also being notably devoid of behavior change content features.
\end{abstract}

Key Words: mobile applications, weight management, behavior change, diet, nutrition (J Nutr Educ Behav. 2021;53:732-741.)

Accepted May 25, 2021. Published online July 24, 2021.

\section{INTRODUCTION}

Obesity continues to be a significant global health challenge. In the US, the prevalence of obesity among adults remains elevated at $42.4 \%{ }^{1}$ Considering the notable comorbidities associated with obesity, ${ }^{2-5}$ the development of affordable and effective treatment modalities remains a paramount focus for public health. Advancements in pharmacological approaches $^{6,7}$ and surgical interventions $^{8}$ have had important impacts on treatment for obesity. However, lifestyle modification through behavior change is still widely recognized as the best approach in terms of accessibility, cost, and effectiveness. ${ }^{9}$

Behavioral approaches to weight management have relatively low implementation costs, but their effectiveness is dependent on participant compliance, which tends to decline over time. ${ }^{10-12}$ However, behavioral interventions supported by information technology have shown promising outcomes. ${ }^{10,13-15}$ These tech-based support systems aid in guiding behavior change related to human health

\footnotetext{
${ }^{1}$ Department of Nutritional Sciences, Rutgers University, New Brunswick, NJ

${ }^{2}$ Department of Human Ecology, Rutgers University, New Brunswick, NJ

Conflict of Interest Disclosure: The authors have not stated any conflicts of interest.

Address for correspondence: Telema Briggs, BS, Department of Nutritional Sciences, Rutgers University, 102 Food Science Bldg, 65 Dudley Rd, New Brunswick, NJ 08901; E-mail: telema.briggs@rutgers.edu

(C) 2021 The Authors. Published by Elsevier Inc. on behalf of Society for Nutrition Education and Behavior. This is an open access article under the CC BY-NC-ND license (http://creativecommons.org/licenses/by-nc-nd/4.0/)

through a broad category of tools known as mobile health (mHealth) technologies. They include specialized portable medical monitoring devices such as wearable heart trackers and multifunctional software tools, such as mobile applications (apps)-software programs capable of running on small wireless computing devices such as smartphones and tablets.

The potential for mHealth apps to enhance behavior modification efforts to improve disease management-related outcomes is well documented in the medical literature. ${ }^{16-18}$ Interventions using smartphone diet and nutrition apps have been shown to assist with behavior change and adoption of healthful habits related to weight management in a research setting. ${ }^{19,20}$ Although prior research has shown behavior change to be a highly effective approach to weight management, studies assessing the utility of nutrition apps have emphasized their lack of evidence-based behavior change 
content. $^{21,22}$ Despite this deficit, which likely limits their potential effectiveness, the use of smartphone nutrition apps among health-conscious consumers continues to increase.

These apps are often used by consumers without the guidance of a registered dietitian nutritionist (RDN) trained in providing and supporting client-centered approaches and treatments to address weight management and other nutrition-related needs. These nutrition professionals can help clients set, review, and maintain realistic goals for their weight and nutritional needs and communicate the intended and unintended consequences of particular strategies. Moreover, they can also add much-needed context to the information collected and provided by an app, preventing misunderstandings that might substantially affect the health of the client (eg, inappropriate interpretation of body mass index).

The consequences and challenges of obesity have been felt disproportionately among low-income individuals. $^{23}$ Although free and trial versions of some weight management apps are available, the inability to afford the advanced features of the paid/upgraded versions of these apps may be a concern, particularly if those versions include evidencebased behavior change content, or might provide the kind of context or information that would likely be communicated by nutrition professionals. Therefore, a comparison of what features are available in free and paid/upgraded versions of the most popular weight management apps may be beneficial, particularly in choosing apps with behavior change features proven to be effective in facilitating successful weight management.

Although many prospective purchasers and users of apps rely on indications of app popularity (ie, ratings and number of installations) to choose an app from among those offered, the relationship between app popularity and the inclusion of evidence-based behavior change functions likely to enhance an app's effectiveness remains undocumented. Thus, this study conducted an analysis of the specific features found in free and paid subscription versions of popular weight management apps and explored the associations between the inclusion of various features (eg, physical activity, dietary intake) and popularity among app users (eg, app ratings). We hypothesized that the total number of features possessed by an app would be strongly associated with user ratings, upgrade costs, and the number of installations.

\section{METHODS}

This study protocol was approved by Rutgers University Institutional Review Board. For inclusion in the analysis, English language versions of nutrition apps needed to be available within both major app distribution platforms, Apple App Store (Apple iOS) and Google Play Store (Android OS). A search for weight management apps conducted on September 10, 2019, using the keywords "diet" and "weight loss" yielded 220 apps.

These inclusion criteria were further refined to select apps with calorie tracking as their primary capability and evidence of at least 500,000 installations, making them among the most frequently downloaded within the app category. Applications were excluded from the study if their overall function was to address primary health outcomes other than weight management (eg, fertility and maternal health) or to address nutrition for the management of specific diseases (eg, diabetes, hypertension, and heart disease). Applications were also eliminated if they were grounded in a single dietary approach (eg, vegan, ketogenic, or gluten-free) or were regionally or culturally specific (eg, apps exclusively emphasizing food intake of Asian Indian cuisine).

\section{Application Features}

The features of the apps observed were organized into 4 categories (dietary intake, anthropometric measurements, physical activity, behavior change strategies) that supported the adult weight management guidelines $^{23}$ developed by the Academy of Nutrition and Dietetics. The dietary intake category referred to features (eg, calorie intake tracker, nutritional database) in an app that assists the user with collecting and recording information from selfmonitored food consumption, such as macronutrients, vitamins, and micronutrients. For organizational purposes, the features within this category were divided into 1 of the 3 subcategories: core features (features assisting with the primary function of nutrition information tracking), macronutrients (eg, carbohydrates and saturated fat), and micronutrients (eg, vitamins and minerals). The anthropometric measurement features included those that record the user's body composition (eg, height, weight, waist circumference), particularly as it relates to determining overweight and obesity status. The physical activity features included those designed to account for the user's caloric expenditures through exercise and purposeful movement (eg, calorie expenditure tracker). The behavior change (BC) strategies features were the app's attempt to implement a variety of evidence-based behavior modification strategies to improve dietary intake and physical activity that include core constructs (eg, self-monitoring). In addition to these core BC strategies, motivational interviewing (MI) techniques (eg, change talk) were also assessed. These techniques serve as client-centered approaches to counseling incorporated by RDNs to generate intrinsic motivators to better facilitate and inspire sustainable behavior change. ${ }^{24,25}$ The coding categories for BC strategies used in this analysis were derived from previous works describing their use in obesity and weight management interventions. $^{26-29}$ Application metadata was also recorded, cataloging additional information about the app, including app rating by users, number of installations, version iteration, and the app's technical capabilities, such as integration with mHealth tracking devices and technologies.

\section{Confirmation of Features}

Free versions of the selected apps were downloaded to electronic touchscreen tablet PC devices (Apple iPad, Apple, Cupertino, CA), which 
was used to evaluate the presence or absence of features specified within each category. However, free versions of Noom (Noom, Inc, New York, NY) and WW (formerly Weight Watchers International, Inc, New York, NY) were unavailable at the time of the investigation. After purchasing a subscription, their features became accessible for evaluation and were included in the data along with the other paid apps. Following this preliminary evaluation, 5 reviewers were recruited and trained to carry out the app evaluation procedures. Training consisted of a data coder presentation detailing study objectives, data collection strategies, and a working demonstration for identifying features of interest within a test app. Reviewers were also given a data coder instruction and terminology manual, which provided further clarification of the study objectives and goals (available on request). After training, reviewers' coding judgments were assessed to ensure interrater reliability of at least $80 \%$ with the lead investigator's initial review of the apps.

All apps were initially reviewed to determine their likely burden of review and equally distributed among trained reviewers. The resulting distribution of apps included a combination of apps with a low, moderate, and high burden of review, each downloaded to iPads. Documentation of the presence or absence of app features was conducted using the following codes: (1) yes if present, (2) no if absent, (3) requires in-app purchase (IAP) if the feature required a subscription before becoming available, or (4) unsure/do not know if none of the previously described responses could be definitively selected on review. All IAP features were documented separately for later review of the upgraded apps. Following the review of the free versions available, app subscriptions were purchased to upgrade apps to their premium or subscription versions. The review of the premium versions of these apps consisted of confirming the availability of the IAP features added through the paid version of the app. Data collected from app reviews were recorded using Qualtrics software (Qualtrics, Provo, UT).

\section{Data Analysis}

Descriptive statistics were used to summarize differences in in-app feature appearance frequencies between free and paid app versions. Agreement between the preliminary app review and the assigned app reviewer's coding for the presence or absence of app features was tested using Cohen's kappa. Discrepancies between reviewer observations were resolved by consensus, resulting in $100 \%$ agreement. Spearman rankorder correlation analyses were conducted to determine the relationships among user app metadata statistics and the features present in the dietary intake, physical activity, BC strategy, and anthropometric tracking categories. All analyses were performed using SPSS (version 26, IBM Corp, Armonk, NY; 2019).

\section{RESULTS}

Fifteen nutrition apps meeting the inclusion criteria were evaluated in February 2020 (Table 1). Less than half of the apps $(n=7,46 \%)$ in the Apple iOS distribution platform were ranked in the health and fitness category by platform app editors. Of these, 1 was selected as an Editor's Choice app in the Apple app platform, whereas 3 were awarded the same designation within the Android/Google app platform. The Editor's Choice designation is defined as an app or game that the app store editors believe are the best in that category or fulfill the purpose of that keyword/category. This, in turn, improves app discoverability and the likelihood of downloading by prospective users. The same apps generated a higher mean 5-star rating $(4.7 \pm 0.09)$ on the Apple iOS platform than the mean 5-star rating for Android versions (4.5 \pm 0.14$)$. The mean rating of nutrition apps across both distribution platforms was 4.6 (SD, 0.08). With the exceptions of WW and Noom, all apps were available for download and provided broad access to app features without the purchase of a subscription. Upgraded subscriptions for all apps were available for both monthly and annual plans, with annual subscription costs ranging from \$29.99 (My
Diet Coach, YAZIO, SparkPeople, and Argus) to $\$ 219.99$ (WW). The mean cost of an annual app subscription was \$63.13 (median, \$44.99) at the time the apps were reviewed. Noom and WW annual subscriptions were considerably more expensive than other apps (\$199.00 and \$219.99, respectively). When excluding these subscriptions, the mean upgrade subscription cost was $\$ 40.63$ (SD, 9.44).

The dietary intake features category was organized into 3 subcategories: core features, macronutrients, and micronutrients (Table 2). Core features describe capabilities that are important components of the primary function of calorie tracking in weight management nutrition apps (ie, nutritional database access, nutrition information). All of the free versions of the apps evaluated $(n=13)$ possessed features essential to calorie tracking, such as a calorie tracking food diary, access to a nutritional database, and a barcode scanner for packaged foods. Twelve of the apps provided descriptions of nutrition information, 11 included energy intake recommendations, 10 had a recipe importer, and 9 had a water consumption tracker.

Features such as food image recognition $(\mathrm{n}=3,23 \%)$ and food intake pattern recognition $(n=6,46 \%)$ infrequently appeared in the 13 free app versions $(n=5)$ but became more available within the 15 upgraded versions $(n=13)$. Among free version apps, a majority $(\mathrm{n}=12,80 \%)$ possessed at least $66 \%$ of the features included in this subcategory. After purchasing a subscription, the average frequency of inclusion of core features was 89\% among all paid apps.

The other 2 subcategories focused on the app's ability to allow the user to personalize targeted macronutrient and micronutrient intake levels to assist in meeting individualized weight management goals (see Table 2). This feature was available in varying capacities in nearly half $(n=7$, $54 \%$ ) of the free versions of the apps and 14 of the 15 upgraded apps. Protein, carbohydrates, and total fat were commonly found to be the most modifiable intake options among apps capable of personalized intake $(n=7,54 \%$ before upgrading; 


\begin{tabular}{|c|c|c|c|c|c|}
\hline $\begin{array}{l}\text { App Name } \\
\text { (Developer or Publisher) }\end{array}$ & Installations & $\begin{array}{l}\text { Apple } \\
\text { Ratings }^{\text {a }}\end{array}$ & $\begin{array}{l}\text { Google } \\
\text { Ratings }^{a}\end{array}$ & $\begin{array}{l}\text { Average } \\
\text { Rating }\end{array}$ & $\begin{array}{l}\text { Annual } \\
\text { Subscription } \\
\text { Cost, \$ }\end{array}$ \\
\hline $\begin{array}{l}\text { MyFitnessPal }{ }^{\mathrm{b}, \mathrm{c}, \mathrm{d}} \\
\text { MyFitnessPal, Inc. }\end{array}$ & $50,000,000$ & 4.7 & 4.5 & 4.6 & 49.99 \\
\hline $\begin{array}{l}\text { Noom }^{c} \\
\text { Noom, Inc }\end{array}$ & $10,000,000$ & 4.7 & 4.1 & 4.4 & 199.00 \\
\hline $\begin{array}{l}\text { Lose } I t^{\mathrm{b}, \mathrm{c}, \mathrm{d}} \\
\text { FitNow, Inc. }\end{array}$ & $10,000,000$ & 4.7 & 4.6 & 4.7 & 39.99 \\
\hline $\begin{array}{l}\text { Lifesum }^{\mathrm{b}, \mathrm{d}, \mathrm{e}} \\
\quad \text { Lifesum, AB }\end{array}$ & $10,000,000$ & 4.6 & 4.4 & 4.5 & 44.99 \\
\hline $\begin{array}{l}\text { WW } \\
\text { Weight Watchers International, Inc. }\end{array}$ & $10,000,000$ & 4.8 & 4.5 & 4.7 & 219.99 \\
\hline $\begin{array}{l}\text { My Diet Coach } \\
\text { Inspired Apps, Mobile Heroes }\end{array}$ & $10,000,000$ & 4.6 & 4.5 & 4.6 & 29.99 \\
\hline $\begin{array}{l}\text { Calorie Counter by Fat Secret } \\
\text { Secret Industries Pty. Ltd. }\end{array}$ & $10,000,000$ & 4.7 & 4.7 & 4.7 & 38.99 \\
\hline $\begin{array}{l}\text { YAZIO_Diet \& Food Tracker } \\
\text { YAZIO, GmbH }\end{array}$ & $10,000,000$ & 4.6 & 4.5 & 4.6 & 29.99 \\
\hline $\begin{array}{l}\text { My Plate Calorie Counter by LIVESTRONG } \\
\text { Leaf Group Ltd. }\end{array}$ & $1,000,000$ & 4.6 & 4.6 & 4.6 & 44.99 \\
\hline $\begin{array}{l}\text { Fooducate } \\
\text { Fooducate, Ltd. }\end{array}$ & $1,000,000$ & 4.7 & 4.4 & 4.6 & 34.99 \\
\hline $\begin{array}{l}\text { MyNetDiary } \\
\text { MyNetDiary.com }\end{array}$ & $1,000,000$ & 4.7 & 4.6 & 4.7 & 59.99 \\
\hline $\begin{array}{l}\text { SparkPeople Calorie Tracker } \\
\text { SparkPeople, Inc. }\end{array}$ & $1,000,000$ & 4.6 & 4.4 & 4.5 & 29.99 \\
\hline $\begin{array}{l}\text { Calorie Carb and Fat Counter by Virtuagym } \\
\text { DigiFit BV }\end{array}$ & $1,000,000$ & 4.8 & 4.5 & 4.7 & 46.99 \\
\hline $\begin{array}{l}\text { Argus } \\
\text { Azumio, Inc. }\end{array}$ & 500,000 & 4.9 & 4.4 & 4.7 & 29.99 \\
\hline $\begin{array}{l}\text { Cronometer }^{\mathrm{b}} \\
\text { Cronometer Software Inc. }\end{array}$ & 500,000 & 4.7 & 4.6 & 4.7 & 46.99 \\
\hline
\end{tabular}

WW indicates Weight Watchers.

${ }^{\mathrm{a}}$ App store ratings as of September 9, 2019; ${ }^{\mathrm{b}}$ Denotes ranked app in Health \& Fitness category of Apple App Store; ${ }^{\mathrm{c}}$ Denotes ranked app in Health \& Fitness category for Google Play Store; 'Denotes Editor's Choice designation for Google Play Store; ${ }^{e}$ Denotes Editor's Choice designation for Apple App Store.

$n=14,93 \%$ postpurchase). The capability to adjust dietary intake amounts for fiber, added sugar, cholesterol, and saturated fat occurred less frequently than other macronutrients. The ability to individualize micronutrient intake goals was generally less common across all free and paid versions of apps, with only a third $(n=5,33 \%)$ of all paid apps being capable of performing this function after upgrading. Individualized micronutrient intakes most frequently included vitamins A, C, and $\mathrm{D}$; sodium; potassium; iron; and calcium. Notably, Cronometer tracked considerably more vitamins $(\mathrm{n}=12$, $92 \%)$ and minerals $(\mathrm{n}=14,78 \%)$ than the other apps assessed (see Table 2).
Most apps track anthropometric measurements as a means to monitor the user's progress toward achieving weight management goals. All of the apps had tools for entering goal weight, as well as current weight, height, age, and gender, as this information is required to set recommendations for individualized calorie intake goals (see Table 3). Other measurements of interest such as waist circumference $(\mathrm{n}=7,47 \%)$, body mass index $(n=5,33 \%)$ and body fat percentage $(n=6,40 \%)$ became more widely available after upgrading apps. Physical activity is also tracked in nutrition/weight management apps as a means to estimate the user's daily calories burned as it relates to total daily energy expenditure. All assessed apps had tools for estimating calorie expenditure through physical activity, capturing data such as type of activity, intensity, and duration (see Table 3). The greatest difference observed among apps within the anthropometric measurement category was in the number of devices with which these apps are capable of integrating (ranging from 1 to 12 devices; median, 6), as it has become increasingly common for apps to sync with smartwatches, smart-scales, and other personal electronic monitoring devices.

Behavior modification is an integral component of weight management interventions and nutrition counseling. The results of the apps' 
Table 2. Presence or Absence of Dietary Intake Features by Smartphone Nutrition App

\section{Category}

Core Features

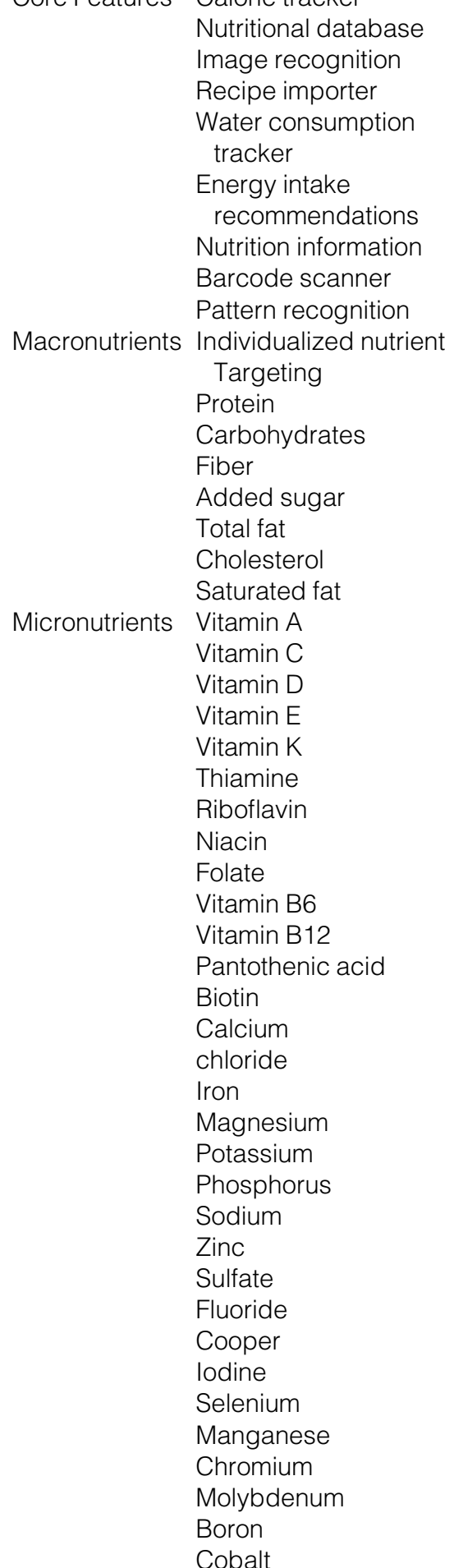

MFP LI Noom ${ }^{a}$ LS WW WW $^{\mathrm{a}}$ MPLC MDS YZ FD MND SP ARG VG CRM

$\begin{array}{lllllllll}\checkmark & \checkmark & \text { IAP } & \checkmark & \text { IAP } & \checkmark & \checkmark & \checkmark & \checkmark \\ \checkmark & \checkmark & \text { IAP } & \checkmark & \text { IAP } & \checkmark & \checkmark & \checkmark & \checkmark \\ - & \checkmark & - & \text { IAP } & - & - & \text { IAP } & \checkmark & - \\ \checkmark & \checkmark & \text { IAP } & \text { IAP } & \text { IAP } & \checkmark & \text { IAP } & \checkmark & \checkmark \\ \checkmark & \text { IAP } & \text { IAP } & \checkmark & \text { IAP } & \checkmark & \text { IAP } & \text { IAP } & \checkmark\end{array}$

$\begin{array}{lllllll}\text { IAP } & \checkmark & \text { IAP } & \checkmark & \text { IAP } & \checkmark & - \\ \text { IAP } & \checkmark & \text { IAP } & \checkmark & \text { IAP } & \checkmark & \checkmark \\ \checkmark & \checkmark & \text { IAP } & \checkmark & \text { IAP } & \checkmark & \checkmark \\ \checkmark & \text { IAP } & \text { IAP } & \text { IAP } & \text { IAP } & \text { IAP } & -\end{array}$

$\begin{array}{ll}\checkmark & \checkmark \\ \checkmark & \sqrt{ } \\ - & - \\ \sqrt{ } & \sqrt{ } \\ \sqrt{ } & \sqrt{ }\end{array}$

$\begin{array}{llll}\checkmark & \checkmark & \checkmark & \checkmark \\ \checkmark & \checkmark & \checkmark & \checkmark \\ - & \checkmark & - & - \\ \text { IAP } & \checkmark & \checkmark & \checkmark \\ \checkmark & \checkmark & - & \checkmark\end{array}$

IAP IAP

IAP IAP

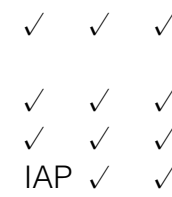

$\begin{array}{llll}\checkmark & \checkmark & \checkmark & \checkmark \\ \checkmark & \checkmark & \checkmark & \checkmark \\ \checkmark & \checkmark & \checkmark & \checkmark \\ \text { IAP } & \checkmark & & \checkmark\end{array}$

$\checkmark \quad$ IAP IAP $\checkmark \checkmark \quad \checkmark \quad$ IAP $\checkmark$

$\checkmark \quad$ IAP IAP

IAP IAP

IAP IAP

IAP IAP

IAP IAP

IAP IAP

IAP IAP

IAP IAP

IAP IAP
IAP IAP

IAP IAP

IAP IAP

IAP IAP

IAP IAP

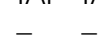

$\begin{array}{ll}- & - \\ - & - \\ - & -\end{array}$

$-$

$-$

$-$ $\checkmark \quad$ IAP IAP $\quad \checkmark \quad \checkmark \quad$ IAP $\checkmark$

$\checkmark \quad$ IAP IAP $\quad \checkmark \quad \checkmark \quad$ IAP $\checkmark$

- IAP IAP $\checkmark \checkmark \checkmark \quad$ IAP $\checkmark$

- IAP IAP $-\checkmark \checkmark$ IAP $\checkmark$

$\checkmark \quad$ IAP IAP $\checkmark v \quad \checkmark \quad$ IAP $\checkmark$

- IAP IAP $\checkmark \checkmark \checkmark \checkmark \quad$ IAP $\checkmark$

$-\quad$ IAP IAP $-\sqrt{ } \quad$ IAP $\checkmark$

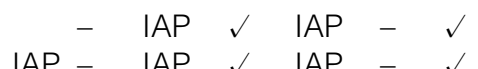$$
\text { IAP - IAP } \checkmark \text { - } \quad-\sqrt{ }
$$$$
\text { IAP - IAP - } \quad \text { I }
$$$$
\text { IAP - IAP }-\quad-\quad-\quad \checkmark
$$

ARG indicates Argus; CRM, Cronometer; FD, Fooducate; FS, Fat Secret; IAP, in-app purchase; LI, Lose It; LS, Lifesum; MDC, My Diet Coach; MFP, MyFitnessPal; MND, MyNetDiary; MPL, MyPlate Livestrong; SP, SparkPeople; VG, Virtuagym; WW, Weight Watchers; YZ, YAZIO.

aUnable to assess without app subscription.

Note: $\checkmark$ denotes presence of feature or function. 
Table 3. Presence or Absence of Anthropometric Measurements and Physical Activity Features in Smartphone Nutrition App

\begin{tabular}{|c|c|c|c|c|c|c|c|c|c|c|c|c|c|c|c|c|}
\hline Category & Feature & MFP & LI & Noom $^{a}$ & LS & $\mathbf{w W}^{\mathbf{a}}$ & MPL & MDC & FS & YZ & FD & MND & SP & ARG & VG & CRM \\
\hline \multirow[t]{14}{*}{ Anthropometrics } & Weight & $\checkmark$ & $\sqrt{ }$ & IAP & $\checkmark$ & IAP & $\checkmark$ & $\checkmark$ & $\checkmark$ & $\checkmark$ & $\checkmark$ & $\checkmark$ & $\checkmark$ & $\sqrt{ }$ & $\checkmark$ & $\checkmark$ \\
\hline & Height & $\checkmark$ & $\sqrt{ }$ & IAP & $\checkmark$ & IAP & $\checkmark$ & $\sqrt{ }$ & $\checkmark$ & $\checkmark$ & $\checkmark$ & $\checkmark$ & $\checkmark$ & $\checkmark$ & $\checkmark$ & $\checkmark$ \\
\hline & Gender & $\checkmark$ & $\sqrt{ }$ & IAP & $\checkmark$ & IAP & $\sqrt{ }$ & $\checkmark$ & $\checkmark$ & $\checkmark$ & $\checkmark$ & $\checkmark$ & $\checkmark$ & $\checkmark$ & $\checkmark$ & $\sqrt{ }$ \\
\hline & Age & $\checkmark$ & $\checkmark$ & IAP & $\checkmark$ & IAP & $\checkmark$ & $\sqrt{ }$ & $\sqrt{ }$ & $\sqrt{ }$ & $\checkmark$ & $\sqrt{ }$ & $\sqrt{ }$ & $\sqrt{ }$ & $\checkmark$ & $\sqrt{ }$ \\
\hline & Waist circumference & $\sqrt{ }$ & IAP & - & $\checkmark$ & - & - & IAP & - & IAP & IAP & - & - & - & $\checkmark$ & \\
\hline & Hip circumference & $\checkmark$ & IAP & - & IAP & - & - & - & - & IAP & - & - & - & - & - & - \\
\hline & Arm circumference & - & IAP & - & IAP & - & - & - & - & IAP & - & - & - & - & - & - \\
\hline & Chest circumference & - & IAP & - & IAP & - & - & - & - & IAP & - & - & - & - & - & - \\
\hline & Neck circumference & - & IAP & - & IAP & - & - & - & - & IAP & - & - & - & - & - & - \\
\hline & Thigh circumference & - & IAP & - & IAP & - & - & - & - & IAP & - & - & - & - & - & - \\
\hline & Body fat, \% & - & IAP & - & IAP & - & - & - & - & IAP & - & - & - & $\checkmark$ & $\checkmark$ & $\sqrt{ }$ \\
\hline & Waist-to-hip ratio & - & & - & IAP & - & - & IAP & - & - & - & - & - & - & - & - \\
\hline & BMI & - & & - & $\checkmark$ & - & $\checkmark$ & $\checkmark$ & - & - & - & - & - & - & $\checkmark$ & $\sqrt{ }$ \\
\hline & Ideal body weight & - & & - & - & - & - & - & - & - & - & - & - & - & - & - \\
\hline \multirow[t]{6}{*}{ Physical Activity } & $\begin{array}{l}\text { Calorie expenditure } \\
\text { tracker }\end{array}$ & $\checkmark$ & $\checkmark$ & IAP & $\checkmark$ & IAP & $\checkmark$ & $\checkmark$ & $\checkmark$ & $\checkmark$ & $\checkmark$ & $\checkmark$ & $\sqrt{ }$ & IAP & IAP & $\checkmark$ \\
\hline & Activity duration & $\sqrt{ }$ & $\sqrt{ }$ & IAP & $\checkmark$ & IAP & $\checkmark$ & $\sqrt{ }$ & $\sqrt{ }$ & $\checkmark$ & $\checkmark$ & $\sqrt{ }$ & $\checkmark$ & IAP & IAP & $\sqrt{ }$ \\
\hline & Activity intensity & $\sqrt{ }$ & $\sqrt{ }$ & IAP & $\checkmark$ & IAP & $\sqrt{ }$ & $\sqrt{ }$ & $\sqrt{ }$ & $\sqrt{ }$ & $\sqrt{ }$ & $\sqrt{ }$ & $\checkmark$ & IAP & IAP & $\sqrt{ }$ \\
\hline & Activity type & $\checkmark$ & $\sqrt{ }$ & IAP & $\checkmark$ & IAP & $\sqrt{ }$ & $\sqrt{ }$ & $\checkmark$ & $\checkmark$ & $\checkmark$ & $\checkmark$ & $\checkmark$ & IAP & IAP & $\sqrt{ }$ \\
\hline & Technology integration & $\checkmark$ & $\checkmark$ & IAP & $\checkmark$ & IAP & $\checkmark$ & $\checkmark$ & $\checkmark$ & $\sqrt{ }$ & $\sqrt{ }$ & $\sqrt{ }$ & $\checkmark$ & IAP & IAP & $\sqrt{ }$ \\
\hline & $\begin{array}{l}\text { Number of integrated } \\
\text { devices }\end{array}$ & 8 & 10 & 10 & 8 & 6 & 2 & 2 & 2 & 3 & 1 & 4 & 6 & 12 & 1 & 7 \\
\hline
\end{tabular}

ARG indicates Argus; BMI, body mass index; CRM, Cronometer; FD, Fooducate; FS, Fat Secret; IAP, in-app purchase; LI, Lose It; LS, Lifesum; MDC, My Diet Coach; MFP, MyFitnessPal; MND, MyNetDiary; MPL, MyPlate Livestrong; SP, SparkPeople; VG, Virtuagym; WW, Weight Watchers; YZ, YAZIO.

aUnable to assess without app subscription.

Note: $\checkmark$ denotes presence of feature or function.

incorporation of evidence-based BC constructs and MI techniques are displayed in Table 4. All 13 of the free versions of the apps and all 15 of the upgraded versions were observed to engage the user in core $\mathrm{BC}$ constructs such as goal setting and self-monitoring. In addition, 11 of the free versions and 13 of the paid versions offered social support and motivational messaging. Structured meal planning/portion control became increasingly available as app functionality was upgraded through subscription purchases, appearing in 6 of the free versions and 13 of the upgraded versions.

The ability of apps to perform and incorporate MI techniques was also assessed as a subcomponent of the BC feature assessment. Frequently observed MI techniques used by apps included providing advice and feedback (found in 11 of the free and 14 of the upgraded versions), normalization ( 8 of the free and 10 of the upgraded versions; 53\%), and affirmations ( $n=5$ of the free and 7 of the upgraded versions). Motivational interviewing-based BC content was visible within the apps in a number of different ways; advice and feedback appeared as detailed nutrition content analysis with suggestions for improvement. Normalizing and affirmations were both frequently observed in user community forums where users were able to share common struggles and successes with weight management (ie, normalizing) and provide support and affirmative words of encouragement (ie, affirmations). Notably, Noom and WW were both observed to possess all assessed core and MI constructs on subscription purchases.

Table 5 shows the results of Spearman rank-order correlations among average app ratings assessed in-app metadata (ie, as a proxy for popularity among consumers) and the features present in dietary intake, physical activity, BC strategy, and anthropometric tracking feature categories. Results of the Spearman correlation indicated that there was a significant positive correlation between average app rating and the group of features controlling for the user's ability to track specific macronutrients (eg, protein, dietary fiber, etc) $\quad\left(r_{\mathrm{s}}=0.74\right.$; $P=0.002)$. The presence of these groups of features was also positively correlated with the total costs of the app subscription $\left(r_{\mathrm{s}}=0.60, P=0.019\right)$. In addition, the inclusion of core $\mathrm{BC}$ strategies in an app was positively correlated with the use of $\mathrm{MI}$ techniques $\left(r_{\mathrm{s}}=0.73, P=0.002\right)$; however, neither the inclusion of core BC strategies nor MI was significantly correlated with app ratings or subscription costs. Finally, there was a positive correlation between the total number of features available in an app and subscription costs $\left(r_{\mathrm{s}}=0.71 ; P=0.003\right)$.

\section{DISCUSSION}

The availability and use of smartphone apps dedicated to nutrient and calorie tracking have increased as consumers seek the use of technology to support weight management. 
Table 4. Presence of Core Behavior Change Constructs and Motivational Interviewing Techniques in Smartphone App

\begin{tabular}{|c|c|c|c|c|c|c|c|c|c|c|c|c|c|c|c|c|}
\hline Category & Feature & MFP & LI & Noom $^{a}$ & LS & $\mathbf{w W}^{\mathbf{a}}$ & MPL & MDC & FS & YZ & FD & MND & $\mathbf{S P}$ & ARG & VG & CRM \\
\hline Core & Self-monitoring & $\sqrt{ }$ & $\sqrt{ }$ & IAP & $\sqrt{ }$ & IAP & $\checkmark$ & $\checkmark$ & $\sqrt{ }$ & $\sqrt{ }$ & $\sqrt{ }$ & $\checkmark$ & $\sqrt{ }$ & $\checkmark$ & $\checkmark$ & $\checkmark$ \\
\hline \multirow[t]{11}{*}{ constructs } & Stress management & $\checkmark$ & $\sqrt{ }$ & IAP & - & IAP & $\sqrt{ }$ & $\sqrt{ }$ & - & - & $\checkmark$ & $\checkmark$ & $\sqrt{ }$ & $\checkmark$ & - & - \\
\hline & Stimulus control & & $\checkmark$ & IAP & - & IAP & $\sqrt{ }$ & $\checkmark$ & - & - & $\checkmark$ & $\checkmark$ & $\checkmark$ & - & - & - \\
\hline & Problem solving & - & - & IAP & - & IAP & - & - & - & - & - & - & - & - & - & - \\
\hline & Contingency management & - & - & IAP & $\sqrt{ }$ & IAP & - & $\checkmark$ & - & - & $\checkmark$ & - & $\sqrt{ }$ & - & - & - \\
\hline & Cognitive restructuring & $\checkmark$ & IAP & IAP & - & IAP & - & $\sqrt{ }$ & - & - & $\checkmark$ & $\sqrt{ }$ & $\sqrt{ }$ & - & - & - \\
\hline & Social support & $\checkmark$ & $\sqrt{ }$ & IAP & $\checkmark$ & IAP & $\checkmark$ & $\checkmark$ & $\sqrt{ }$ & $\checkmark$ & $\checkmark$ & $\sqrt{ }$ & $\sqrt{ }$ & $\sqrt{ }$ & - & - \\
\hline & $\begin{array}{l}\text { Structured meal plan/ } \\
\text { portion control }\end{array}$ & $\checkmark$ & IAP & IAP & IAP & IAP & $\sqrt{ }$ & - & IAP & $\sqrt{ }$ & IAP & $\checkmark$ & - & $\checkmark$ & $\sqrt{ }$ & IAP \\
\hline & Relapse prevention & - & - & IAP & - & IAP & - & $\sqrt{ }$ & - & - & - & - & - & - & - & - \\
\hline & Motivational messaging & $\checkmark$ & $\checkmark$ & IAP & $\checkmark$ & IAP & $\checkmark$ & $\checkmark$ & $\sqrt{ }$ & - & $\checkmark$ & $\sqrt{ }$ & $\checkmark$ & $\sqrt{ }$ & $\sqrt{ }$ & - \\
\hline & Goal setting & $\checkmark$ & $\sqrt{ }$ & IAP & $\sqrt{ }$ & IAP & $\checkmark$ & $\checkmark$ & $\checkmark$ & $\checkmark$ & $\checkmark$ & $\checkmark$ & $\checkmark$ & $\sqrt{ }$ & $\sqrt{ }$ & $\checkmark$ \\
\hline & Goal progression & $\checkmark$ & $\sqrt{ }$ & IAP & $\sqrt{ }$ & IAP & $\checkmark$ & - & - & $\checkmark$ & $\checkmark$ & $\sqrt{ }$ & $\checkmark$ & $\sqrt{ }$ & $\checkmark$ & $\sqrt{ }$ \\
\hline \multirow{11}{*}{$\begin{array}{c}\text { Motivational } \\
\text { interviewing } \\
\text { techniques }\end{array}$} & Change talk & - & - & IAP & & IAP & - & - & - & - & - & - & - & - & - & - \\
\hline & Open-ended questioning & - & - & IAP & & IAP & - & - & - & - & - & - & - & - & - & - \\
\hline & Reflective listening & - & - & IAP & & IAP & - & - & - & - & - & - & - & - & - & - \\
\hline & Decision balancing & - & - & IAP & & IAP & - & - & - & - & - & - & - & - & - & - \\
\hline & Affirmations & $\checkmark$ & $\sqrt{ }$ & IAP & & IAP & - & $\checkmark$ & $\sqrt{ }$ & - & - & - & $\sqrt{ }$ & - & - & - \\
\hline & Normalizing & $\checkmark$ & $\sqrt{ }$ & IAP & & IAP & $\checkmark$ & $\checkmark$ & $\sqrt{ }$ & - & $\checkmark$ & $\sqrt{ }$ & $\sqrt{ }$ & - & - & - \\
\hline & $\begin{array}{l}\text { Assessing readiness } \\
\text { of change }\end{array}$ & - & - & IAP & - & IAP & - & - & - & - & - & - & - & - & - & - \\
\hline & Advice/feedback & $\checkmark$ & IAP & IAP & $\checkmark$ & IAP & $\checkmark$ & $\checkmark$ & $\sqrt{ }$ & - & $\checkmark$ & $\checkmark$ & $\sqrt{ }$ & $\sqrt{ }$ & $\checkmark$ & $\checkmark$ \\
\hline & $\begin{array}{l}\text { Exploration of importance } \\
\text { and confidence }\end{array}$ & - & - & IAP & - & IAP & - & - & - & - & - & - & - & - & - & - \\
\hline & Summaries (in relation to) & - & - & IAP & - & IAP & - & - & - & - & - & - & - & - & - & - \\
\hline & $\begin{array}{l}\text { Statements supporting } \\
\text { self-efficacy }\end{array}$ & - & - & IAP & - & IAP & - & $\checkmark$ & - & - & - & - & - & - & - & - \\
\hline
\end{tabular}

ARG indicates Argus; CRM, Cronometer; FD, Fooducate; FS, Fat Secret; IAP, in-app purchase; LI, Lose It; LS, Lifesum; MDC, My Diet Coach; MFP, MyFitnessPal; MND, MyNetDiary; MPL, MyPlate Livestrong; SP, SparkPeople; VG, Virtuagym; WW, Weight Watchers; YZ, YAZIO.

a Unable to assess without app subscription.

Note: $\checkmark$ denotes presence of feature or function.

\section{Table 5. Nutrition Smartphone App Features and Metadata: Correlation Matrix}

\begin{tabular}{|c|c|c|c|c|c|c|c|c|c|c|c|}
\hline Variables & 1 & 2 & 3 & 4 & 5 & 6 & 7 & 8 & 9 & 10 & 11 \\
\hline 1. App ratings & - & & & & & & & & & & \\
\hline 2. Total features & 0.186 & - & & & & & & & & & \\
\hline 3. Subscription cost & 0.399 & $0.711^{\star \star}$ & - & & & & & & & & \\
\hline 4. Total installations & -0.236 & 0.101 & 0.185 & - & & & & & & & \\
\hline 5. Core features & -0.003 & 0.255 & 0.437 & 0.276 & - & & & & & & \\
\hline 6. Macronutrients & $0.740^{* \star}$ & 0.465 & $0.598^{\star}$ & -0.059 & 0.392 & - & & & & & \\
\hline 7. Vitamins & -0.088 & 0.404 & 0.100 & -0.284 & -0.043 & -0.119 & - & & & & \\
\hline 8. Minerals & -0.020 & 0.124 & 0.083 & -0.213 & 0.182 & 0.003 & 0.478 & - & & & \\
\hline 9. Anthropometrics & -0.308 & -0.202 & -0.116 & 0.190 & 0.043 & -0.184 & -0.058 & -0.197 & - & & \\
\hline 10. BC Core strategies & 0.073 & 0.289 & 0.231 & 0.243 & -0.103 & 0.028 & -0.313 & -0.407 & -0.443 & - & \\
\hline 11. BC Ml techniques & 0.047 & 0.205 & 0.199 & 0.494 & -0.100 & -0.002 & -0.420 & -0.099 & -0.479 & $0.725^{* *}$ & - \\
\hline
\end{tabular}

$\mathrm{BC}$ indicated behavior change; MI, modivational interviewing.

${ }^{\star}$ Correlation is significant at the 0.05 level (2-tailed); ${ }^{\star \star}$ Correlation is significant at the 0.01 level (2-tailed).

Note: Descriptive statistics $(n=15)$; Correlations are Spearman Rank Order $\left(r_{s}\right)$. 
In this study, the free and paid versions of 15 popular nutrition smartphone apps were analyzed to identify their features defined in the areas of dietary intake, anthropometric measurement, physical activity, and BC. The study also examined associations between the presence of specific feature categories and metadata attributes such as app rating, subscription cost, and the number of installations. Of the hypothesized associations between metadata and the overall total number of app features, only app subscription cost was found to be positively correlated with the number of features. In addition, the study confirmed that most nutrition apps possess an abundance of features dedicated to dietary intake, anthropometric, and physical activity tracking while also being notably devoid of BC content features.

Several studies have performed assessments of features in smartphone nutrition apps for weight management in varying capacities. These assessments are wide-ranging and include evaluating the use of emergent technology in dietary assessment strategies ${ }^{30-32}$ and approaches for quantifying and appraising an app's potential to facilitate BC. ${ }^{21,33,34}$ This study contributes to the growing body of literature that describes the capabilities of nutrition apps. Findings from this study suggested that the inclusion of $\mathrm{BC}$ features did not seem to be a significant factor in influencing average app ratings, cost, or the number of installations. This was true despite including apps such as Noom and WW, which have been heavily promoted as a tool for BC. Moreover, the apps identified as Editor's Choice (ie, My Fitness Pal, Lose It, and Life Sum) all lacked features employing core BC constructs and MI techniques, even after upgrading to their paid versions. The lack of association between the inclusion of $\mathrm{BC}$ features and average app rating or app subscription cost may further suggest that consumers do not recognize the importance of $\mathrm{BC}$ content when considering the selection of an app. These findings are concerning given the research showing that using $\mathrm{BC}$ strategies can lead to significantly better health outcomes as it relates to weight management. ${ }^{35-37}$
Interestingly, the strongest correlation was observed between average app rating and the ability to adjust macronutrient intake. This finding is consistent with a recent study that suggests that self-monitoring of food intake through tracking, independent of dietary approach, leads to better weight loss outcomes in overweight individuals. ${ }^{38}$ However, other attributes not assessed, such as user interface, aesthetics, usability, and user experience, are known to affect user engagement with apps and exert a strong influence on app ratings as well. ${ }^{22}$ Therefore, it is unlikely that macronutrient tracking is the only significant driver of app ratings. In addition, this finding may suggest a general desire for consumers to individualize their macronutrient intake to accommodate specific dietary approaches such as ketogenic, low-carb, or high protein diets. The consumer demand for control of individualized diets has become more apparent as the number of apps dedicated to specific dietary approaches and dietary restrictions continues to grow within the digital marketplace.

Although this study offers meaningful contributions to the literature, there are several limitations. First, there are significant differences in how Apple and Google present app metrics, such as user ratings and number of installations to consumers. For instance, Apple allows app developers to reset the collection of app ratings as new iterations are released on its platform. However, developers are cautioned to use rating resets sparingly, as not to discourage potential users from downloading an app because of too few ratings. Thus, ratings are more likely to reflect multiple or possibly all app iterations. ${ }^{39}$ However, the ratings on the Google Play Store appear to give greater consideration to the latest version of an app and are weighted toward the most recent reviews. ${ }^{40}$ Furthermore, when considering app ratings, it is important to note that user ratings, though mostly indicative of the app itself, occasionally speak to user satisfaction with an app distribution platform at large. ${ }^{41}$ Although Android devices are more widely distributed globally, Apple has become increasingly competitive and currently dominates the smartphone operating system market. $^{42}$ This has translated to increased consumer exposure to the iPhone and can potentially account for some differences in ratings for apps across platforms.

Discerning the full scope of $\mathrm{BC}$ content was also challenging because some apps require relatively large amounts of user input before employing behavior change strategies. This was apparent in apps, such as Lose It, which required users to $\log$ in dietary intake information for 7 consecutive days before BC content became available. The app reviewers for this study used the apps for only 2 days, and thus, the full scope of BC content may not have been fully captured during this period of assessment.

\section{IMPLICATIONS FOR RESEARCH AND PRACTICE}

Most free or trial versions of weight management apps possess a number of basic, essential features that assist users with tracking and monitoring various aspects of dietary intake and physical activity. These in-app capabilities are enhanced to an extent after purchasing app subscriptions. These improvements appear to be primarily related to enhancing dietary intake tracking in an effort to influence progress with weight management goals. Although most lack key BC features, these apps are capable of supporting BC systems ${ }^{43}$ as they all have features that can assist users with goal setting and strategies like self-monitoring, crucial components to weight management BC. In addition, these apps may also be capable of serving in a limited capacity as useful extensions of professional dietetic practice by enhancing specific components of the nutrition care process $^{44}$ such as nutrition assessment (the systematic collection of client's nutrition-related information) and nutrition monitoring/evaluation (used to determine the amount of progress made by a client from a nutrition intervention).

This study and others have demonstrated that the ability of diet and nutrition apps to fully employ $\mathrm{BC}$ 
strategies remains inadequate. However, although these current nutrition apps are incapable of replacing $\mathrm{RDN} /$ nutrition professionals, the majority of individuals who elect to use nutrition apps do so without the guidance of a trained professional. ${ }^{45}$ There are significant opportunities for future research to not only explore ways in which these tools can optimize approaches to clientbased weight management interventions but also to foster innovative collaborations between RDN/nutrition professionals and app developers to create nutrition apps better suited to support patients and clients in the management of nutrition-related needs.

\section{ACKNOWLEDGMENTS}

The authors would like to thank the research assistants for serving as app reviewers and participating in the data collection necessary to perform this content analysis.

\section{REFERENCES}

1. Hales CM, Carroll MD, Fryar CD, Ogden CL. Prevalence of obesity and severe obesity among adults: United States, 2017-2018. NCHS Data Brief. 2020:(360):1-8.

2. Wolf AM, Colditz GA. Current estimates of the economic cost of obesity in the United States. Obes Res. 1998;6:97-106.

3. Wolf AM. What is the economic case for treating obesity? Obes Res. 1998;6 (suppl 1):2S-7S.

4. Finkelstein EA, Trogdon JG, Cohen JW, Dietz W. Annual medical spending attributable to obesity: payer- and service-specific estimates. Health Aff. 2009;28(suppl 1):w822-w831.

5. Trogdon J, Finkelstein EA, Hylands T, Dellea PS, Kamal-Bahl S. Indirect costs of obesity: a review of the current literature. Obes Rev. 2008;9:489-500.

6. Jain SS, Ramanand SJ, Ramanand JB, Akat PB, Patwardhan MH, Joshi SR. Evaluation of efficacy and safety of orlistat in obese patients. Indian J Endocrinol Metab. 2011;15:99.

7. Fidler MC, Sanchez M, Raether B, et al. A one-year randomized trial of lorcaserin for weight loss in obese and overweight adults: the BLOSSOM trial. J Clin Endocrinol Metab. 2011;96:3067-3077.

8. Lysen L, Israel D. Nutrition in weight management. In: Mahan LK, Raymond JL, editors. Krause's Food \& the Nutrition Care Process. 14th ed. Elsevier; 2017: 383-406.

9. Van Dorsten B, Lindley EM. Cognitive and behavioral approaches in the treatment of obesity. Med Clin North Am. 2011;95:971-988.

10. Klasnja P, Consolvo S, McDonald DW, Landay JA, Pratt W. Using mobile \& personal sensing technologies to support health behavior change in everyday life: lessons learned. AMIA Annu Symp Proc. 2009;2009:338-342.

11. World health organization. Adherence to Long-Term Therapies: Evidence for Action. World Health Organization; 2003.

12. Kelley CP, Sbrocco G, Sbrocco T. Behavioral modification for the management of obesity. Prim Care Clin Off Pract. 2016;43:159-175

13. Khaylis A, Yiaslas T, Bergstrom J, Gore-Felton C. A review of efficacious technology-based weight-loss interventions: five key components. Telemed J E Health. 2010;16:931-938.

14. Neve M, Morgan PJ, Jones P, Collins C. Effectiveness of web-based interventions in achieving weight loss and weight loss maintenance in overweight and obese adults: a systematic review with meta-analysis. Obes Rev. 2010;11:306-321.

15. Norman GJ, Zabinski MF, Adams MA, Rosenberg DE, Yaroch AL, Atienza AA. A review of eHealth interventions for physical activity and dietary behavior change. $A m$ J Prev Med. 2007;33:336-345.

16. Bhuyan SS, Lu N, Chandak A, et al. Use of mobile health applications for health-seeking behavior among US adults. J Med Syst. 2016;40:153.

17. Patel MS, Asch DA, Volpp KG. Wearable devices as facilitators, not drivers, of health behavior change. JAMA. 2015;313:459-460.

18. Free C, Phillips G, Felix L, Galli L, Patel V, Edwards P. The effectiveness of $\mathrm{m}$-health technologies for improving health and health services: a systematic review protocol. BMC Res Notes. 2010;3:250.

19. Turner-McGrievy GM, Beets MW, Moore JB, Kaczynski AT, Barr-Anderson DJ, Tate DF. Comparison of traditional versus mobile app self- monitoring of physical activity and dietary intake among overweight adults participating in an mHealth weight loss program. J Am Med Inform Assoc. 2013;20:513-518.

20. Carter MC, Burley VJ, Nykjaer C, Cade JE. Adherence to a smartphone application for weight loss compared to website and paper diary: pilot randomized controlled trial. J Med Internet Res. 2013;15:e32.

21. Azar KM, Lesser LI, Laing BY, et al. Mobile applications for weight management: theory-based content analysis. Am J Prev Med. 2013;45:583589.

22. Bardus M, van Beurden SB, Smith JR, Abraham C. A review and content analysis of engagement, functionality, aesthetics, information quality, and change techniques in the most popular commercial apps for weight management. Int $J$ Behav Nutr Phys Act. 2016;13:35.

23. Academy of Nutrition and Dietetics. Adult weight management (AWM) guideline (2014). https://www.andeal. $\mathrm{org} / \mathrm{topic}$. cfm? $\mathrm{menu}=52768 \mathrm{cat}=4688$. Accessed September 9, 2019.

24. Miller WR, Rollnick S. Motivational Interviewing: Helping People Change. Guilford Press; 2012.

25. Hettema J, Steele J, Miller WR. Motivational interviewing. Annu Rev Clin Psychol. 2005;1:91-111.

26. NHLBI obesity education initiative. The Practical Guide: Identification, Evaluation, and Treatment of Overweight and Obesity in Adults. US Department of Health and Human Services. Public Health Service, National Institutes of Health, National Heart, Lung and Blood Institute; 2000.

27. Spahn JM, Reeves RS, Keim KS, et al. State of the evidence regarding behavior change theories and strategies in nutrition counseling to facilitate health and food behavior change. J Am Diet Assoc. 2010;110:879-891.

28. Riley WT, Rivera DE, Atienza AA, Nilsen W, Allison SM, Mermelstein R. Health behavior models in the age of mobile interventions: are our theories up to the task? Transl Behav Med. 2011;1:53-71.

29. West JH, Hall PC, Arredondo V, Berrett B, Guerra B, Farrell J. Health behavior theories in diet apps. J Con Health Internet. 2013;17:10-24.

30. Franco RZ, Fallaize R, Lovegrove JA, Hwang F. Popular nutrition-related 
mobile apps: a feature assessment. JMIR MHealth UHealth. 2016;4:e85.

31. Lieffers JR, Hanning RM. Dietary assessment and self-monitoring: with nutrition applications for mobile devices. Can J Diet Pract Res. 2012;73:e253-e260.

32. Jake-Schoffman DE, Silfee VJ, Waring ME, et al. Methods for evaluating the content, usability, and efficacy of commercial mobile health apps. JMIR mHealth uHealth. 2017;5:e190.

33. Chen J, Cade JE, Allman-Farinelli M. The most popular smartphone apps for weight loss: a quality assessment. JMIR mHealth uHealth. 2015;3:e104.

34. Pagoto S, Schneider K, Jojic M, DeBiasse M, Mann D. Evidence-based strategies in weight-loss mobile apps. Am J Prev Med. 2013;45:576-582.

35. Teixeira PJ, Marques MM. Health behavior change for obesity management. Obes Facts. 2017;10:666-673.

36. Kahan S. Overweight and obesity management strategies. Am J Manag Care. 2016;22(7 Suppl):s186-s196.

37. LeBlanc ES, Patnode CD, Webber EM, Redmond N, Rushkin M, O'Connor
EA. Behavioral and pharmacotherapy weight loss interventions to prevent obesity-related morbidity and mortality in adults: updated evidence report and systematic review for the US Preventive Services Task Force. JAMA. 2018;320:1172-1191.

38. Patel ML, Hopkins CM, Brooks TL, Bennett GG. Comparing self-monitoring strategies for weight loss in a smartphone app: randomized controlled trial. JMIR mHealth uHealth. 2019;7:e12209.

39. Apple Inc. Ratings, Reviews and Responses - App Store. https://developer. apple.com/app-store/ratings-and-reviews/\#: :text $=$ Ratings $\% 20$ and $\% 20$ reviews $\% 20$ influence $\% 20$ how, of $\% 20$ $1 \% 20$ to $\% 205 \% 20$ stars. \&text $=$ This $\%$ 20 summary\%20rating \%20is\%20specific,new\%20version\%20of\%20your \%20app. Accessed August 21, 2020.

40. Nguyen G. New app rating formula headed to Google Play will favor newer reviews. https://searchengineland.com/ new-app-rating-formula-headed-to-google-play-316697. Accessed August 21, 2020.
41. App Radar. How to get users to leave an app store rating. https://appradar. $\mathrm{com} / \mathrm{blog} /$ ask-users-leave-review-inapp-stores. Accessed January 9, 2021.

42. Statista. Subscriber share held by smartphone operating systems in the United States from 2012 to 2020. https:/ / www. statista.com/statistics/266572/marketshare-held-by-smartphone-platforms-inthe-united-states/. Accessed January 8 , 2021.

43. Oinas-Kukkonen H, Harjumaa M. Persuasive systems design: key issues, process model, and system features. Commun Assoc Inf Syst. 2009;24:28.

44. Swan WI, Vivanti A, Hakel-Smith NA, et al. Nutrition Care Process and Model update: toward realizing peoplecentered care and outcomes management. J Acad Nutr Diet. 2017;117: 2003-2014.

45. Lieffers JR, Vance VA, Hanning RM. Use of mobile device applications in Canadian dietetic practice. Can J Diet Pract Res. 2014;75:41-47.

\title{
Thank you to
}

\section{Leslie Cunningham-Sabo, PhD, RDN Colorado State University}

\author{
for completing her term \\ on JNEB's Board of Editors
}

